\title{
Probing the optical readout characteristics of Fabry-Perot ultrasound sensors through realistic modelling
}

Dylan M. Marques, James A. Guggenheim, Rehman Ansari, Edward Z. Zhang, Paul C. Beard, et al.

Dylan M. Marques, James A. Guggenheim, Rehman Ansari, Edward Z. Zhang, Paul C. Beard, Peter R. T. Munro, "Probing the optical readout characteristics of Fabry-Perot ultrasound sensors through realistic modelling," Proc. SPIE 11077, Opto-Acoustic Methods and Applications in Biophotonics IV, 110770H (19 July 2019); doi: 10.1117/12.2527114 


\title{
Probing the optical readout characteristics of Fabry-Pérot ultrasound sensors through realistic modelling
}

\author{
Dylan M. Marques, James A. Guggenheim, Rehman Ansari, Edward Z. Zhang, Paul C. Beard, \\ and Peter R. T. Munro \\ Department of Medical Physics and Biomedical Engineering, University College London, \\ London, U.K.
}

\begin{abstract}
The Fabry-Pérot interferometer (FPI) is widely used in photoacoustic imaging (PAI) as an ultrasound (US) sensor due to its high sensitivity to weak US waves. Such high sensitivity is important as it allows for increasing the depth in tissue at which PAI can access, thus strongly influencing its clinical applicability. FPI sensitivity is impacted by many factors including the FPI mirror reflectivity, focussed beam spot size, FPI cavity thickness and aberrations introduced by the optical readout system. Improving FPI sensitivity requires a mathematical model of its optical response which takes all of these factors into account. Previous attempts to construct such a model have been critically limited by unrealistic assumptions. In this work we have developed a general model of FPI optical readout which based upon electromagnetic theory. By making very few assumptions, the model is able to replicate experimental results and allows insight to be gained into the operating principles of the sensor.
\end{abstract}

Keywords: Photoacoustic imaging, Electromagnetic modelling, Fabry-Perot interferometer

\section{INTRODUCTION}

Fabry-Pérot ultrasound sensors have been used extensively to produce high resolution in-vivo photoacoustic images of, for example, mouse ear vasculature, ${ }^{1}$ genetically labelled xenografts implanted in mouse ${ }^{2}$ and mouse embryos. ${ }^{3}$ An FPI can be used as an US sensor since its optical reflectivity is highly sensitive to the ratio of cavity thickness to optical wavelength. ${ }^{4}$ A fundamental assumption when using an FPI as a US sensor is that US waves perturb the cavity thickness, thus leading to a change in its optical reflectivity. Consider, for example, an FPI composed of mirrors with reflectivity of $98 \%$ and a cavity thickness of $204.75 \mu \mathrm{m}$, the wavelength dependent reflectivity profile for a plane wave at normal incidence is shown in Fig. 1. Note, however, that the reflectivity profile is very sensitive to the angle of incidence of the plane wave. This is also shown in Fig. 1 which shows that changing the plane wave's angle of incidence to 0.01 radians results in a significant shift in the reflectivity profile. This is an important observation, since plane waves cannot be used to obtain a localised measurement of cavity thickness due to their infinite spatial extent. Such a measurement is obtained using a focussed beam, resulting in an interferometer transfer function (ITF), a typical example of which is plotted in Fig. 1, which has a vastly different shape to that of a plane wave. Note that the method of field detection, i.e., fiber or photodiode in free space, has a significant influence on the ITF as is also shown in Fig. 1, which we discuss later.

\section{MODELLING THE INTERFEROMETER TRANSFER FUNCTION}

We begin by noting that the sensitivity of FPI US sensors depends on both the acoustic and optical sensitivity and that here we focus on the optical sensitivity. The ITFs in Fig. 1 are calculated using a realistic model based upon electromagnetic theory. In particular, we consider the Debye-Wolf diffraction integral ${ }^{6}$ which is a very general expression that relates the field in the focal region of a, potentially high numerical aperture, objective lens to the field incident upon its aperture. The Debye-Wolf integral is outlined in Fig. 2 which can be interpreted as representing the field in the focal region as a superposition of plane waves. Importantly, each point within the lens aperture gives rise to one, and only one, such plane wave. The complex amplitude of each

Further author information:

Peter Munro: E-mail: p.munro@ucl.ac.uk

Opto-Acoustic Methods and Applications in Biophotonics IV, edited by Vasilis Ntziachristos,

Roger Zemp, Proc. of SPIE-OSA Vol. 11077, 110770H · C 2019 SPIE-OSA

CCC code: $1605-7422 / 19 / \$ 21 \cdot$ doi: $10.1117 / 12.2527114$

Proc. of SPIE-OSA Vol. 11077 110770H-1 

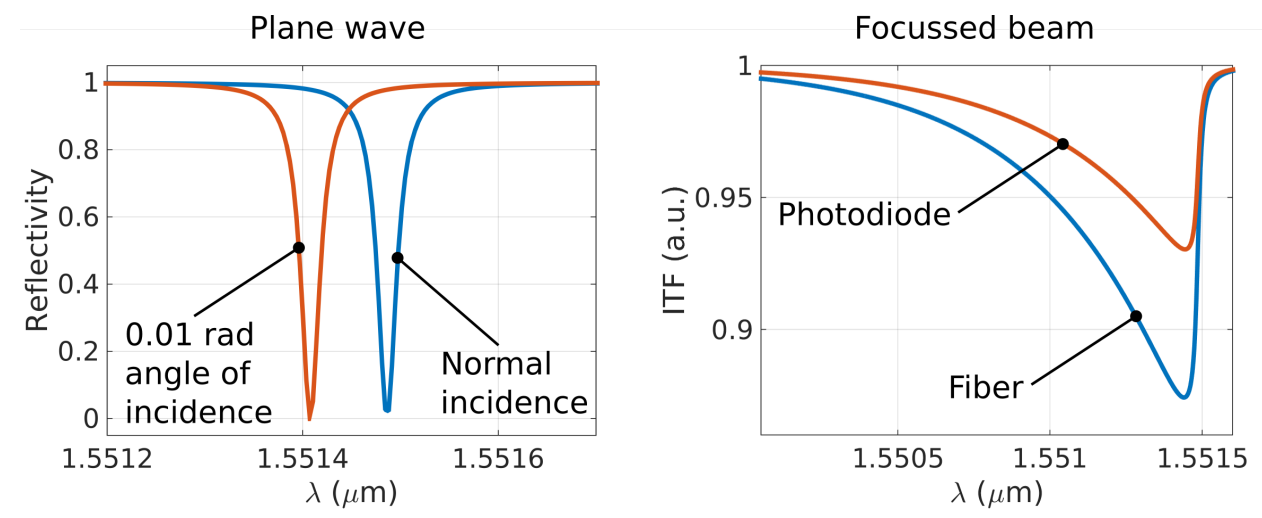

Figure 1. The reflectivity of an FPI for waves at normal and non-normal incidence (left) along with the ITF of an FPI acquired using a focussed beam of $1 / e^{2}$ radius $15 \mu \mathrm{m}$ (right).

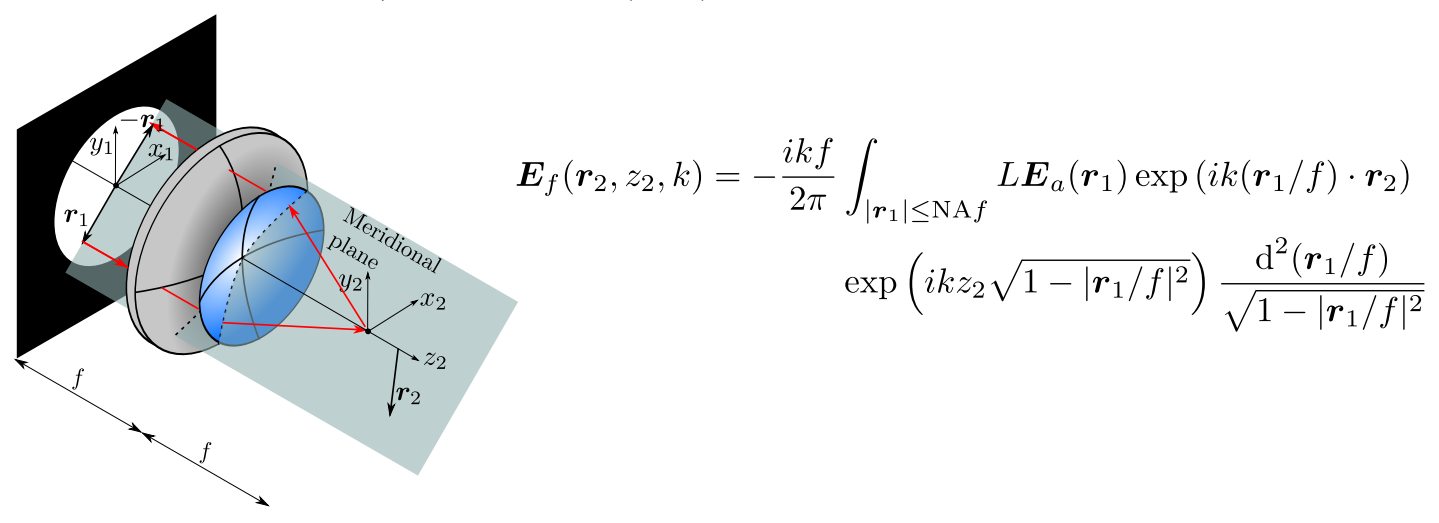

Figure 2. The Debye-Wolf integral (right) along with the coordinate systems it employs (left). $\boldsymbol{E}_{f}$ and $\boldsymbol{E}_{a}$ are the electric fields in the focal region and aperture, respectively. $L$ is a matrix which represents refraction by the lens. ${ }^{5}$

plane wave component is directly proportional to the field incident upon the corresponding location within the aperture. We have, hitherto, considered only the focussing of light into a homogeneous medium. However, it is relatively straightforward to calculate how each plane wave is reflected by the FPI using a matrix formalism for stratified media, ${ }^{7}$ and so it is therefore also straightforward to calculate the electric field, associated with the reflected focussed beam, in the focal region. The ITF could be obtained by calculating the form of the beam reflected by the FPI in the focal region for a range of wavelengths. A more computationally efficient and physically insightful approach is to recognise that when the FPI is aligned normal to the optical axis, as is usually the case, the reflected field at each point, $\boldsymbol{r}_{1}$, within aperture of the objective is related to the field incident upon the aperture at location $-\boldsymbol{r}_{1}$. Another way of saying this is that a ray that enters the aperture at location $\boldsymbol{r}_{1}$ will exit the aperture at location $-\boldsymbol{r}_{1}$ as is shown in Fig. 2. This can be formalised by the expression $\boldsymbol{E}_{a}^{r e f}\left(\boldsymbol{r}_{2}, k\right)=\mathcal{R}\left(\boldsymbol{r}_{2}, k\right) \boldsymbol{E}_{a}^{i n c}\left(\boldsymbol{r}_{2}, k\right)$ where $\boldsymbol{E}_{a}^{\text {ref }}$ and $\boldsymbol{E}_{a}^{\text {inc }}$ are the reflected and incident fields, respectively, in the aperture, $\mathcal{R}$ is a complex term which takes into account reflection by the FPI and the axial location of the FPI and $k$ denotes the wavenumber.

Once $\boldsymbol{E}_{a}^{\text {ref }}$ is obtained, the detector signal can be calculated. If detection in free-space is performed using, for example, a photodiode, the detector signal will be proportional to $I_{1}(k)=\int_{\left|\boldsymbol{r}_{2}\right| \leq f \mathrm{NA}}\left|\mathcal{R}\left(\boldsymbol{r}_{2}, k\right)\right|^{2}\left|\boldsymbol{E}_{a}^{i n c}\left(\boldsymbol{r}_{2}, k\right)\right|^{2} \mathrm{~d}^{2} \boldsymbol{r}_{1}$ whereas if detection is performed using the same fiber used to deliver the incident light, the detected signal will be proportional to $I_{2}(k)=\left|\int_{\left|\boldsymbol{r}_{2}\right| \leq f \mathrm{NA}} \mathcal{R}\left(\boldsymbol{r}_{2}, k\right)\left(\boldsymbol{E}_{a}^{i n c}\left(\boldsymbol{r}_{2}, k\right) \cdot \boldsymbol{E}_{a}^{i n c}\left(\boldsymbol{r}_{2}, k\right)\right) \mathrm{d}^{2} \boldsymbol{r}_{1}\right|^{2}$. We expect these two detection schemes to result in different ITFs, as is confirmed by the ITFs simulated in Fig. 1 using each of the two detection schemes. These simulations confirm what is known from experimental results, that ITFs acquired with tightly focussed beams suffer a loss in visibility and sensitivity. The model can also be used to investigate the nature of the ITF obtained when focussed illumination is employed. Fig. 3a) shows the magnitude of $\boldsymbol{E}_{a}^{r e f}$ at 
three different points (denoted A, B and C) on the same fiber detection ITF plotted in Fig. 1. The reflected field associated with point A on the ITF (i.e., the trough) contains a ring where the reflected field magnitude has a local minimum. Point B on the ITF, which is where the FPI would usually be biased when performing US detection, corresponds to a slightly longer wavelength than point A. The reflected field in the aperture now appears as a spot of local minimum. The ring of local minimum corresponding to point $\mathrm{A}$ is seen to converge closer to the center of the aperture and becomes spot-like. This convergence may be understood by recalling from Fig. 1 that increasing (reducing) the angle of incidence of a plane wave leads to a shift towards smaller (larger) wavelengths of the reflectivity profile. Thus, for a plane wave with angle of incidence $\theta$, if the wavelength is increased by a small amount, the reflectivity of the plane wave can be maintained by reducing its angle of incidence by a corresponding amount. However, the plane wave angle of incidence is related to the position, $\boldsymbol{r}_{1}$ (see Fig. 2) as $\sin \theta=\left|\boldsymbol{r}_{1} / f\right|$ and so increasing the the wavelength causes the reflected field in the aperture to converge towards the centre of the aperture. Increasing the wavelength further to point $\mathrm{C}$ on the ITF causes the local minimum to disappear entirely from the reflected field distribution.
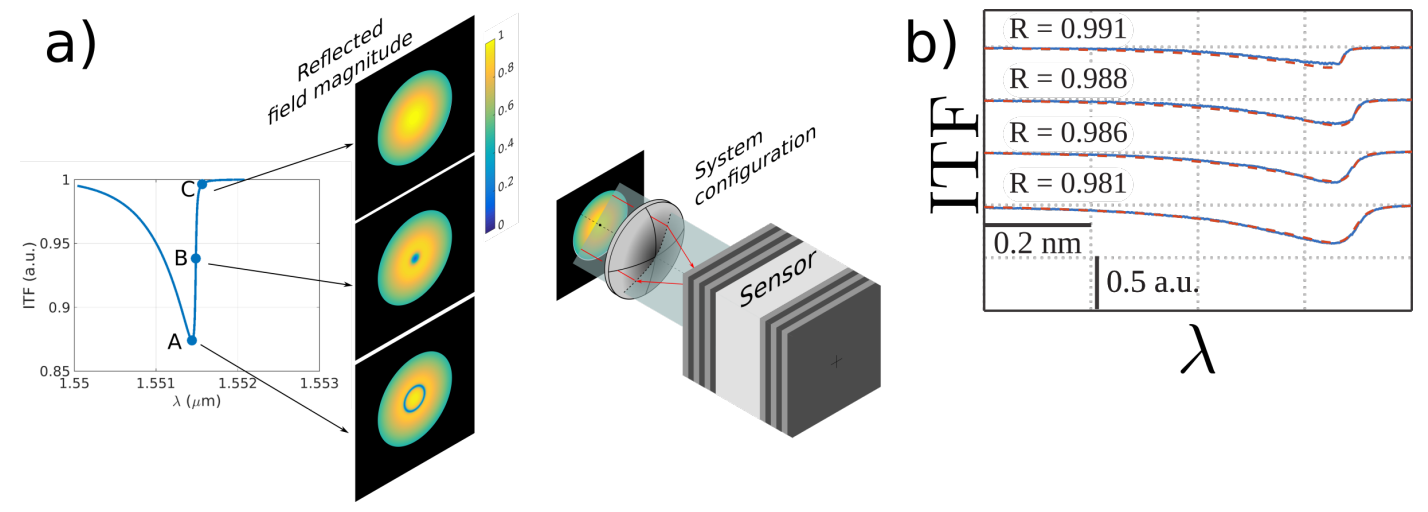

Figure 3. a) The magnitude of reflected fields in the aperture, corresponding to three points in the plotted ITF; b) A comparison between simulated (dashed lines) and experimentally acquired (solid lines) ITFs for a range of mirror reflectivities. A focussed beam of $15 \mu 1 / e^{2}$ radius was employed.

\section{EXPERIMENTAL VALIDATION}

A key aspect of this work is its experimental validation. Fig. 3b) shows an example of the validation that has been performed. It shows a comparison between simulated and experimentally acquired ITFs, for a range of mirror reflectivities and a focussed spot with $1 / e^{2}$ radius of $15 \mu \mathrm{m}$. We have validated the model experimentally for a range of focal spot sizes and in a transmission geometry for the case of free space detection with a photodiode.

\section{CONCLUSION}

We have developed a highly realistic model of FPI ultrasound sensors that agrees well with experimentally acquired ITFs. The model will be useful for optimising the design of FPIs used currently in photoacoustic imaging in order to improve both imaging depth and contrast. We also plan to adapt the model to treat non-planar sensors, such as the recently published planoconcave sensors. ${ }^{1}$

\section{REFERENCES}

[1] J. A Guggenheim et al., Nat. Photonics 11, 714 (2017);

[2] A. P. Jathoul et al., Nat. Photonics 9, 239 (2015).

[3] J. Laufer et al., J. Biomed. Opt. 17, 061220 (2012).

[4] Zhang, E., Laufer, J., and Beard, P., Appl. Optics 47, 561-577 (2008).

[5] Török, P. and Munro, P., Opt. Express 12, 3605-3617 (2004).

[6] Richards, B. and Wolf, E., Proc. R. Soc. Lond. A 253, 358-379 (1959).

[7] Bashara, N., [Ellipsometry and polarized light], North Holland (1987). 